\title{
Estudo comparativo entre sistemáticas de digitalização de documentos: formatos HTML e PDF
}

\section{André Raabe Omer PohImann Filho}

\section{Resumo}

Este artigo apresenta o resultado de experimentos realizados pelo Laboratório de Biblioteca Digital da PUCRS voltados para a captura e conversão de documentos a partir do formato tradicional (papel) para o formato digital. São apresentadas e avaliadas as principais etapas envolvidas no processo de digitalização utilizando duas sistemáticas diferentes: uma baseada na conversão para HTML; a outra baseada na geração de arquivos PDF usados pelo software Adobe Acrobat Reader.

São abordados também fatores essenciais aos trabalhos de digitalização tais como tecnologias de Reconhecimento Óptico dos Caracteres (OCR) e avaliação das

características do acervo a ser digitalizado. Por fim, é realizado um comparativo entre as duas sistemática estudadas, apontando pontos positivos e negativos que devem ser considerados na escolha de uma diretriz de trabalho.

\section{Palavras-chave}

Conversão de documentos do formato tradicional para o digital; Sistemáticas de conversão para HTML; Geração de arquivos PDF; Tecnologias de reconhecimento óptico dos caracteres.
INTRODUÇÃO

A Pontifícia Universidade Católica do Rio Grande do Sul (PUCRS), mediante convênio com a IBM, participa do projeto IBM Global Campus, que prevê a colaboração entre instituições de ensino superior de diferentes países, no sentido de pesquisar e desenvolver políticas, abordagens, metodologias e recursos tecnológicos para projetar e implantar universidades com campus de abrangência global.

A proposta de trabalho do projeto Campus Global PUCRS visa a desenvolver estudos sobre universidade virtual, centrando seu foco de atenção em pesquisas sobre metodologias e recursos tecnológicos na área de educação à distância. Neste contexto, trabalha-se com o conceito de Educação à Distância (EAD), como uma forma de educação na qual alunos e professores se encontram separados fisicamente, sendo o processo de interação multidirecional, apoiado por tecnologia de comunicação, em que o aluno é o protagonista de seu aprendizado e o professor um facilitador deste.

Tendo em vista esta proposta, o projeto Campus Global foi estruturado a partir de quatro frentes de pesquisa, a saber, educação à distância e colaborativa, bibliotecas digitais, trabalho cooperativo, gerência de recursos Internet.

Neste contexto, o Laboratório de Biblioteca Digital vem pesquisando o desenvolvimento de tecnologias para permitir o acesso a informações de conteúdo bibliográfico à distância. Uma das alternativas pesquisadas aponta para a digitalização de documentos e sua disponibilização por meio da Internet.

Para tanto, o Laboratório de Biblioteca Digital voltou-se inicialmente para a pesquisa de software e desenvolvimento de sistemáticas para a captura e transformação de documentos do formato tradicional (papel), para o formato digital. Foram avaliadas duas sistemáticas distintas para realização do trabalho, uma delas baseada no reconhecimento ótico dos caracteres e conversão para HTML detalhada em (Pohlmann ${ }^{1}$ ); a outra baseada no formato digital Portable Document Format (PDF).

\section{DIRETRIZES DE TRABALHO}

Dentre as alternativas pesquisadas para o processo de digitalização de documentos, avaliaram-se duas diretrizes genéricas:

1. digitalização da obra como imagens e conversão destas em textos mediante reconhecimento óptico de caracteres (OCR);

2. criação de arquivos de imagens (JPG), contendo as páginas da obra e mantendo o leiaute original da publicação, sem conversão para texto.

A escolha do formato de arquivo JPG deveu-se ao fato de este possuir uma alta taxa de compressão, permitindo o armazenamento de imagens com qualidade em arquivos de tamanhos reduzidos, sendo, por este motivo, amplamente utilizado na rede Internet. 
Para exemplificar a relação entre formato de arquivo e espaço de armazenagem, foi realizado um teste comparativo permitindo verificar a relação entre os tamanhos dos arquivos gerados no contexto estudado - digitalização de documentos a partir do formato papel. É importante salientar que foram utilizadas rigorosamente as mesmas configurações de compressão e qualidade disponíveis em formatos de arquivos como o JPG e GIF.

No teste, foram utilizadas uma página do livro História da PUCRS ilustrada na figura 1, por esta ser composta de imagem e texto sem cores, e a capa da mesma obra, por ser colorida, figura 2.

A tabela 1 apresenta, a seguir, os resultados do teste comparativo considerando os formatos de arquivos de imagens mais utilizados.

Para avaliar as vantagens e desvantagens relacionadas a cada uma das diretrizes estudadas (conversão para texto e disponibilização como imagem), tomou-se como base (Haigh ${ }^{2}$ ), que considera, para a escolha do processo de digitalização, os seguintes pontos:

- necessidade de reutilização, edição ou reformatação do texto;

- disponibilidade do texto para pesquisas full-text ;

- posterior codificação do texto no formato HTML;

- recursos disponíveis para realização do processo;

- tamanho dos arquivos para armazenagem e transmissão.

Pela análise realizada, chegou-se às seguintes conclusões sobre cada um dos processos:

\section{- Com conversão para texto:}

- possibilidade de edição e manipulação do texto das obras;

- possibilidade de realização de pesquisas full-text,

- processo de digitalização é demorado e trabalhoso;

\section{FIGURA 1}

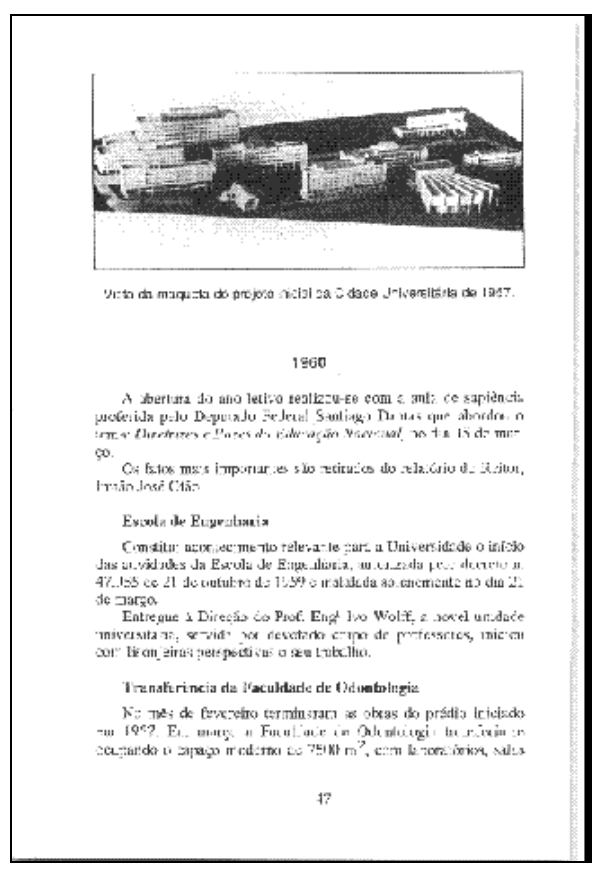

Páginas utilizada como teste comparativo

\section{TABELA 1}

\section{Comparativo entre os tamanhos de arquivo}

Formato do Arquivo

Pagina $P \& B$

Capa (reduzida)

$11,50 \mathrm{~cm} \times 17,80 \mathrm{~cm}$

$7,14 \mathrm{~cm} \mathrm{X} \mathrm{10,68} \mathrm{cm}$

JPG (Joint Picture Experts Group)

TIF (Tagged Image File)

GIF (Graphics Interchange Format)

PDF (Portable Document Format)

PSD (PhotoShop)

PCX (Zsoft Paintbrush)

BMP (Windows Bitmap)

WMF (Windows Meta File)

EPS (Encapsuled Post Script)

- necessita pouco espaço para armazenagem das obras digitalizadas.

\section{- Disponibilização como imagem:}

- impossibilidade de edição e manipulação do texto das obras;

- impossibilidade de realização de pesquisas full-text ;

- processo de digitalização simplificado e rápido;

- ocupa grande espaço para armazenagem da obra digitalizada (aproximadamente 20 vezes mais que textos).
FIGURA 2

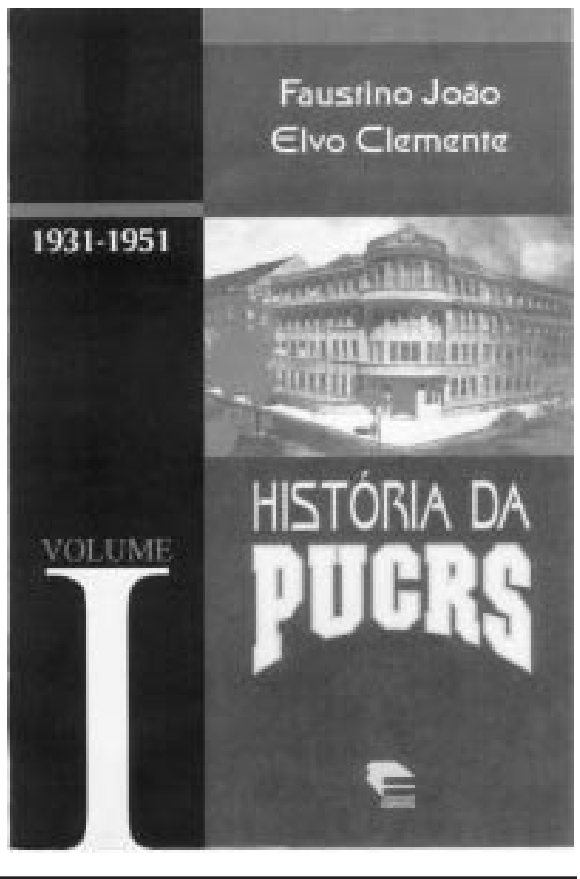

.

$\begin{array}{cc}34 \mathrm{~Kb} & 16 \mathrm{~Kb} \\ 74 \mathrm{~Kb} & 43 \mathrm{~Kb} \\ 76 \mathrm{~Kb} & 40 \mathrm{~Kb} \\ 76 \mathrm{~Kb} & 34 \mathrm{~Kb} \\ 82 \mathrm{~Kb} & 62 \mathrm{~Kb} \\ 97 \mathrm{~Kb} & 64 \mathrm{~Kb} \\ 280 \mathrm{~Kb} & 60 \mathrm{~Kb} \\ 282 \mathrm{~Kb} & 61 \mathrm{~Kb} \\ 573 \mathrm{~Kb} & 135 \mathrm{~Kb}\end{array}$

No contexto geral do projeto, a realização de pesquisa full-text se faz necessária e é um objetivo a ser alcançado. Além disso, outro fator determinante a favor da conversão para texto é que a velocidade de transmissão de dados no Brasil ainda não atinge os padrões desejados para transferência de arquivos de imagem. No caso dos arquivos texto, a velocidade de transmissão não é um fator crítico, pois estes possuem tamanho bem inferior. 
Portanto, decidiu-se proceder inicialmente à digitalização de obras mediante o reconhecimento ótico dos caracteres $(\mathrm{OCR})$ e posterior transformação no formato HTML.

\section{SISTEMÁTICA DE DIGITALIZAÇÃO HTML}

Para realização dos trabalhos voltados à definição de uma sistemática de captura e conversão de documentos para o formato HTML, foi utilizado como instrumento de testes a publicação da Faculdade de Medicina da PUCRS denominada Acta Médica Volume 1. Os trabalhos foram realizados mediante os recursos disponíveis, ou seja, scanner HP Scanjet II, software de OCR (Reconhecimento Ótico de Caracteres) OmniPage Pro 5.0, editor de textos MS-Word 6.0 e o conjunto de softwares do Netscape Communicator 4.0 (Pohlmann $\left.{ }^{1}\right)$.

Inicialmente, são apresentadas as principais características das Actas Médicas, para que se possa ter uma idéia do contexto estudado e da adequação dos recursos utilizados.

Como um compilado de artigos de alunos (trabalhos de conclusão), estas publicações começaram a ser editadas em 1977. Em sua maioria, são documentos antigos que precisam passar pela função de criação e captura, ou seja, conforme (Pohlmann ${ }^{1}$ ) são documentos que têm de ser necessariamente digitalizados.

O objeto inicial desta pesquisa foi a primeira edição da Acta Médica, editada em 1977. Este documento possui um leiaute de texto complexo contendo:

- texto dividido em duas colunas;

- tamanho de letra muito pequeno (aproximadamente tamanho times new roman 10$)$;

- fórmulas matemáticas manuscritas em meio ao texto;

- seqüência de texto não linear (em alguns capítulos, o texto inicia pela coluna da direita);

- figuras e imagens;
FIGURA 3

Representação Esquemática do Processo de Digitalização HTML

Digitalização do texto e Transformação em arquivo H T M L
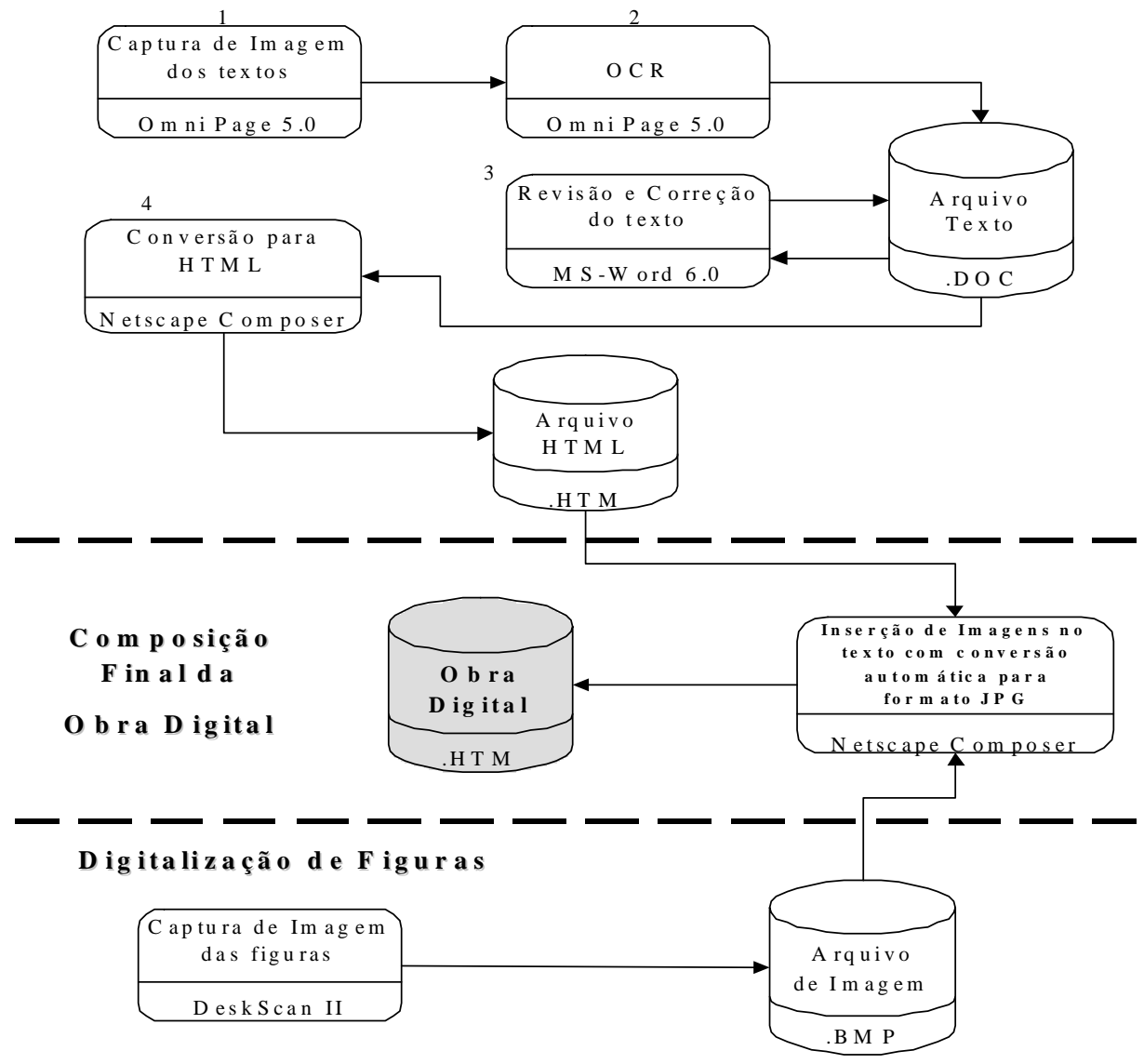

- notas de rodapé;

4) conversão para formato HTML.

- subdivisão de itens por meio de chaves;

- manchas de tinta e falhas na impressão;

- textos e figuras somente na cor preta ou tons de cinza.

\section{Descrição do Processo de Digitalização}

O objetivo do processo foi a transformação da obra para formato digital e sua publicação em formato HTML. Para tanto, dividiu-se o trabalho em quatro etapas:

1) leitura ótica das páginas da obra;

2) reconhecimento do texto por meio de software de OCR;

3) revisão e correção do texto por intermédio de editor de texto; (MS-Word6.0);
A descrição detalhada de cada uma destas etapas pode ser vista no endereço

\section{http://www.cglobal.pucrs.br/ bibdigital/artigos/art3.htm.}

Para cada capítulo do livro, foi criado um arquivo HTML diferente para facilitar a posterior ligação com indexadores, hiperlinks e softwares de gerência de bibliotecas.

O espaço total em disco, ocupado pelos 16 capítulos digitalizados, contendo 241 páginas de texto e 72 figuras, foi de 2,41 megabytes, comprovando eficiência em termos de economia de espaço de armazenagem e conseqüente agilidade no acesso aos documentos full-text, via Internet.

Um resumo deste processo é apresentado, acima, esquematicamente, na figura 3 . 
Na tabela 2 são apresentados também os tempos médios verificados na execução de cada uma destas etapas. Os tempos apresentados são para um número padrão de 50 páginas e 12 figuras.

\section{Busca de melhores resultados no OCR}

A elaboração da sistemática HTML foi feita com a utilização do software de OCR Omni Page Pro 5.0. Os resultados deste experimento apontaram um tempo total de conversão muito alto, principalmente pela necessidade de realização de uma revisão e correção meticulosa dos erros gerados pelo processo de reconhecimento óptico dos caracteres (OCR).

Dando continuidade ao trabalho, desejava-se verificar a utilização de uma versão mais atual do software, o Omni Page Pro 8.0, a fim de identificar meIhorias no processo de reconhecimento de caracteres que reduzissem o trabalho de revisão e correção a patamares aceitáveis, dentro do escopo de um projeto de digitalização em larga escala.

Para realização deste trabalho comparativo, escolheu-se um informativo de publicação interna na PUCRS chamado "PUCRS Informação". O mesmo foi escolhido por possuir uma diagramação elaborada, com fotos e textos distribuídos de forma não-linear, permitindo a comparação entre os procedimentos de definição automática das zonas de texto de ambas as versões do OmniPage Pro.

O processo de definição de zonas de texto pode ser realizado de forma manual, conforme descrito em (Pohlmann ${ }^{1}$ ), produzindo um resultado melhor em termos de fidelidade ao leiaute da obra original, no entanto esta atividade envolve muita interação do usuário tornando o processo lento. A utilização da definição automática das zonas do texto é uma tentativa de reproduzir o leiaute da obra original sem a interação do usuário. No entanto, os resultados ficam aquém dos esperados.

TABELA 2

Tempos médios para realização das etapas

ETAPAS

TEMPO MÉDIO

Captura das imagens dos textos e execução

do programa de reconhecimento ótico de

65 minutos

caracteres - OCR (com a criação do arquivo texto)

400 minutos

Revisão e correção do texto

15 minutos

Conversão dos arquivos texto para arquivos HTML

20 minutos

Captura de imagens e criação de arquivos BMP

10 minutos

Inserção de imagens no texto e composição final da obra

510 minutos

Tempo médio para transformação de um texto de 50 páginas, com 12 figuras, do formato convencional (em papel), para o formato digital, segundo a sistemática proposta

(aprox. 8,5 horas)

Observação: Cumpre salientar que estes tempos foram estimados contando com a participação de duas pessoas para sua realização. Obviamente, quanto maior a equipe, menor o tempo consumido. Também os recursos de hardware utilizados, principalmente o scanner que não possuía recurso ADF (Automatic Document Feeder), não são os recomendados para este tipo de trabalho. A utilização de recursos mais apropriados tende a melhorar as performances observadas, principalmente nas etapas de captura de imagens, revisão e correção de texto, que são críticas neste processo.

Outra característica a ser salientada é a alta qualidade (qualidade laser) de impressão do informativo, bem como a utilização de fontes padrão (arial), o que, segundo (Caere ${ }^{3}$ ), levaria o Omni Page Pro 8.0 a atingir uma taxa de acerto no reconhecimento dos caracteres superior a $99 \%$.

O "PUCRS Informação", composto de 20 páginas, foi digitalizado e armazenado como imagem para posterior reconhecimento dos caracteres e zonas de texto em ambas as versões do OmniPage. Para tanto, foi utilizado o Omni Page Pro 5.0 e um scanner de mesas HP Scanjet II, gerando um arquivo de saída no formato proprietário MET contendo as 20 páginas digitalizadas. Este arquivo foi aberto em ambas as versões 5.0 e 8.0, onde foi realizado o reconhecimento óptico dos caracteres (OCR) e a definição automática das zonas de texto, uma vez que ambas as versões possuem esses recursos.
Após realizado o processo, os arquivos de saída contento o texto reconhecido pelo OCR foram salvos no formato DOC do MS-Word 6.0, por ser comum a ambas as versões e permitir a utilização de um dicionário ortográfico comum na detecção dos erros de reconhecimento dos caracteres. A comparação entre as taxas de reconhecimento atingidas pelas versões 5.0 e 8.0 do Omnipage Pro partiu de uma análise destes arquivos.

Realizou-se a contagem do número total de palavras na obra. A seguir, realizou-se a contagem das palavras que possuíam incorreções na grafia originadas por um erro no reconhecimento dos caracteres. De posse deste valores, calculou-se o percentual de acertos atingido pelo reconhecimento dos caracteres em ambas as versões. Cumpre salientar que os dados obtidos relacionados à taxa de reconhecimento do processo de OCR consideraram as 20 páginas do informativo na íntegra. A tabela 3 , a seguir, ilustra os resultados obtidos. 
Concluiu-se que a utilização de uma versão mais atual do software Omni Page Pro não promoveu significativa melhoria nos resultados do processo de reconhecimento dos caracteres que pudesse acelerar significativamente os trabalhos de digitalização de um acervo em larga escala, uma vez que a necessidade de revisão do texto permaneceu necessária.

\section{Diretrizes para busca de uma nova sistemática}

Segundo $\left(\right.$ Haigh $\left.^{2}\right)$, a taxa de reconhecimento de um OCR para conversão de documentos deve ser superior a $98 \%$. Caso contrário, é mais eficiente realizar a redigitação do documento.

Esta taxa de reconhecimento é medida considerando o número de edições necessárias (inserções, deleções, substituições) diante do número total de caracteres. Recomenda-se, no entanto, que este dado não seja utilizado como referência para trabalhos de digitalização em larga escala, pois desconsidera todo o trabalho de localização de erros no texto, que muitas vezes demanda uma leitura completa da obra. Além disso, quando o vocabulário utilizado é eminentemente técnico, pode ser necessária a confrontação com a obra original em papel, para identificação da grafia correta de uma palavra.

O que deve ser considerado efetivamente é o volume de tempo despendido por um usuário, ao realizar a correção/conferência de um texto reconhecido pelo OCR.

Enquanto os software de OCR não atingirem uma taxa de reconhecimento de $100 \%$, será necessária meticulosa revisão da obra para localização e correção dos erros, atividade essa que torna a realização de trabalhos de digitalização em larga escala altamente custosos, sendo necessária a utilização de grandes equipes com numerosos recursos para que o trabalho não se torne excessivamente demorado.

TABELA 3 Comparativo dos resultados do OCR

\begin{tabular}{lccc}
\hline & Total de palavras & Palavras com erro & $\begin{array}{c}\text { Taxa de } \\
\text { reconhecimento }\end{array}$ \\
\hline Versão 5.0 & $4833^{*}$ & 308 & $93,6 \%$ \\
Versão 8.0 & 4785 & 168 & $96,5 \%$ \\
\hline
\end{tabular}

* A diferença observada no número total de palavras deve-se ao fato de a versão 5.0 dividir algumas palavras ao meio, gerando duas novas.

A integração de dicionários ortográficos ao processo de reconhecimento dos caracteres, como o procede o OmniPage Pro, auxilia a identificação das palavras consideradas suspeitas. No entanto, dada a impossibilidade de se construir um dicionário eletrônico que abranja todos os termos técnicos específicos de cada área, nos diversos idiomas contemplados pelo acervo da biblioteca da PUCRS, persiste a necessidade de realização de uma leitura cuidadosa, pois uma palavra assinalada como suspeita pode estar correta ou não.

Como um agravante, muitas das obras do acervo da Biblioteca Central da PUCRS (aproximadamente 300 mil obras) não apresentam um estado de conservação adequado à realização da digitalização por meio de softwares de OCR, tais como:

- obras com páginas riscadas e com anotações a lápis e a caneta;

- obras com papéis com gramatura muito fina $\left(50 \mathrm{gr} / \mathrm{m}^{2}\right)$, fazendo com que 0 texto de um lado da página seja visível do outro lado;

- obras com páginas amassadas, manchadas, sujas, deterioradas por mofo, traças, ou mesmo pela própria utilização.

Apesar de possuir um setor específico para recuperação do acervo, muitos dos problemas são irrecuperáveis, e, com uma movimentação diária de 2600 empréstimos, a tendência é que tais problemas não sejam eliminados.
Aliado a estes fatores, pode-se mencionar também o fato de que a maioria das obras é composta não somente de textos. Também contêm figuras, fórmulas e esquemas gráficos cujos softwares de OCR atuais não oferecem um tratamento adequado, sendo necessária a utilização de um outro software para captura das imagens e posterior integração. Tal procedimento exige muita interação do usuário e torna o tempo de digitalização de uma obra muito alto, considerando a amplitude do trabalho desejado.

Tais informações levaram os pesquisadores do laboratório de biblioteca digital da PUCRS a buscar outras alternativas de digitalização que envolvessem menor interação do usuário, viabilizando a realização do trabalho em larga escala.

Desta forma, iniciou-se o trabalho de definição de uma nova sistemática de digitalização utilizando o software da empresa Adobe, denominado Adobe Acrobat. Este software foi cedido pela empresa ao Laboratório de Biblioteca Digital da PUCRS para a realização de testes por um período de 60 dias. Segundo $\left(\right.$ Adobe $\left.^{4}\right)$, tem como características:

- facilidade de criação e publicação de documentos on-line;

- mantém o leiaute original das obras digitalizadas;

- utiliza o formato de arquivo PDF (Portable Document Format), que permite a criação de documentos multiplataforma que podem ser visualizados inclusive em browsers (software de navegação na Internet); 
- possibilidade de captura e conversão de grandes volumes de documentos com um baixo nível de interação do usuário.

Estas características se mostraram bastante adequadas ao tipo de trabaIho que se tinha para realizar.

\section{SISTEMÁTICA PDF}

\section{Digitalização por meio da captura de documentos}

Para a definição de uma sistemática de digitalização por meio da captura de documentos utilizando o software Adobe Acrobat, escolheu-se, como objeto de teste, a obra história da PUCRS. Esta escolha deveu-se a adequação da obra ao trabalho que estava proposto e pela liberação dos direitos autorais da obra por parte dos autores, permitindo aliar sua disponibilização à comemoração do cinqüentenário da universidade, ocorrida em 1998, época em que esta pesquisa estava em andamento.

A obra possui somente uma coluna de texto, as letras são Times New Roman de tamanho 12. São encontradas fotografias e ilustrações em meio ao texto, no entanto nenhuma utiliza cores, somente tons de cinza.

Para digitalização da História da PUCRS, foi utilizado o scanner HP Network Scanner 5, destacando-se pela velocidade de digitalização e pela presença de uma bandeja para entrada automática de papel ADF (Automatic Document Feeder), apesar de suportar somente a digitalização de imagens em tons de cinza, fato que não atrapalhou a definição da sistemática.

Para a inserção das páginas da obras na bandeja de entrada de papel, foi necessário realizar um corte rente a parte onde as folhas estão presas para que estas se soltassem. Ao final, a aplicação cliente do scannergera automaticamente um arquivo contendo as páginas digitalizadas como imagens, ou seja, uma imagem para cada página, agrupadas em um só arquivo PDF.

TABELA 4

Tempo para digitalização das obras utilizando o Scanner HP Network 5

\begin{tabular}{lll}
\hline Volume 1 & 159 páginas & 16 minutos \\
Volume 2 & 295 páginas & 36 minutos \\
Total & 454 páginas & 52 minutos
\end{tabular}

Média: 8,73 páginas por minuto

O tempo levado para digitalização dos dois volumes da História da PUCRS está explicitado na tabela 4.

Um dos objetivos previstos era a possibilidade de realização de pesquisas full-text na obra. Para tanto, seria necessário que as imagens digitalizadas passassem por um processo de reconhecimento ótico de caracteres (OCR), ou seja, a transformação da imagem em texto.

A realização de OCR em arquivos no formato PDF é feita pelo software Adobe Acrobat Exchange, o qual possui uma interface adequada para a tarefa, sendo possível a realização do processo sobre todas as páginas de uma vez só.

No entanto, foi frustrante descobrir que o software em sua versão 3.01 não possui dicionário para reconhecimento das palavras e caracteres da língua portuguesa, o que inviabilizou a realização do OCR e, por conseqüência, a transformação da imagem capturada via scanner em um texto.

Apesar disso, duas características interessantes relacionadas ao OCR aplicado pelo Acrobat Exchange devem ser ressaltadas:

1) as palavras ou conjuntos de caracteres não reconhecidos foram mantidos como imagens, de forma que a leitura do texto continuou sendo possível;
2) quando da aplicação do OCR nas páginas da obra, os arquivos reduziram o seu tamanho em aproximadamente quatro vezes;

Dado a inviabilidade de realização do OCR nas páginas da História da PUCRS, alguns dos objetivos traçados no início da pesquisa precisaram ser abandonados. Em especial a possibilidade de realização de pesquisas no texto desta obra.

Neste momento, decidiu-se dar seqüência ao trabalho de digitalização mantendo as páginas da obra como imagens, pois julgou-se importante avaliar as características e o comportamento dos arquivos PDF contendo imagens das páginas digitalizadas.

Partiu-se, então, para a exploração de recursos do formato PDF que permitiriam aprimorar a navegabilidade e aparência da obra, tais como a criação de links e bookmarks, a separação dos capítulos, a adequação do tamanho e da visualização das páginas da obra. Estes ajustes foram realizados utilizando o Adobe Acrobat Exchange. Uma descrição detalhada deste procedimentos pode ser encontrada em http: \\www.cglobal.pucrs.br bibdigital\kits $\backslash k i t 3 . h t m l$. 
A seguir, será apresentado o resumo esquemático do trabalho de digitalização da História da PUCRS (figura 4).

São apresentados também os tempos médios verificados na execução de cada uma destas etapas. Os tempos apresentados correspondem à digitalização do primeiro volume da obra, com 159 páginas (tabela 5).

\section{Digitalização e da conversão de documentos}

Tendo em vista a inadequação do OCR, decidiu-se pesquisar alternativas que tornassem possível a realização de pesquisas full-text. Uma possibilidade encontrada foi a conversão de documentos já existentes no formato digital para o formato PDF, evitando, dessa forma, a necessidade de reconhecimento óptico dos caracteres, uma vez que o documento original não é gerado mediante a captura de imagens com uso de um scanner.

Para a realização deste novo trabalho, foi escolhido como instrumento de testes a dissertação de mestrado do professor Omer Pohlmann Filho, co-autor deste artigo, pela facilidade de negociação dos direitos autorais e pela adequação da obra ao trabalho proposto.

A dissertação em questão consiste de um conjunto de arquivos digitais com 247 páginas divididos entre textos elaborados no Word 2.0 contendo grande número de tabelas, fórmulas e figuras, e oito tabelas elaboradas no Excel 3.0. Pelas características das tabelas, seria muito trabalhoso realizar a conversão para HTML, uma vez que os recursos de tabela disponíveis na linguagem não são satisfatórios para tanto.

O trabalho de conversão consistiu na carga destes arquivos, por meio da utilização de versões mais recentes do Word e do Excel, e posterior impressão dos mesmos utilizando o driver de impressão denominado Adobe PDF Writer. Este driver se encarrega de capturar a impressão e convertê-la para um arquivo PDF.

Foi necessário agrupar estes arquivos em um só, baseando-se na ordenação encontrada na publicação original (em papel).
Figura 4

Esquema de digitalização da história da PUCRS

\section{Digitalização da histónia da PUCRS}

Captura e manipulação das imagens

1

2

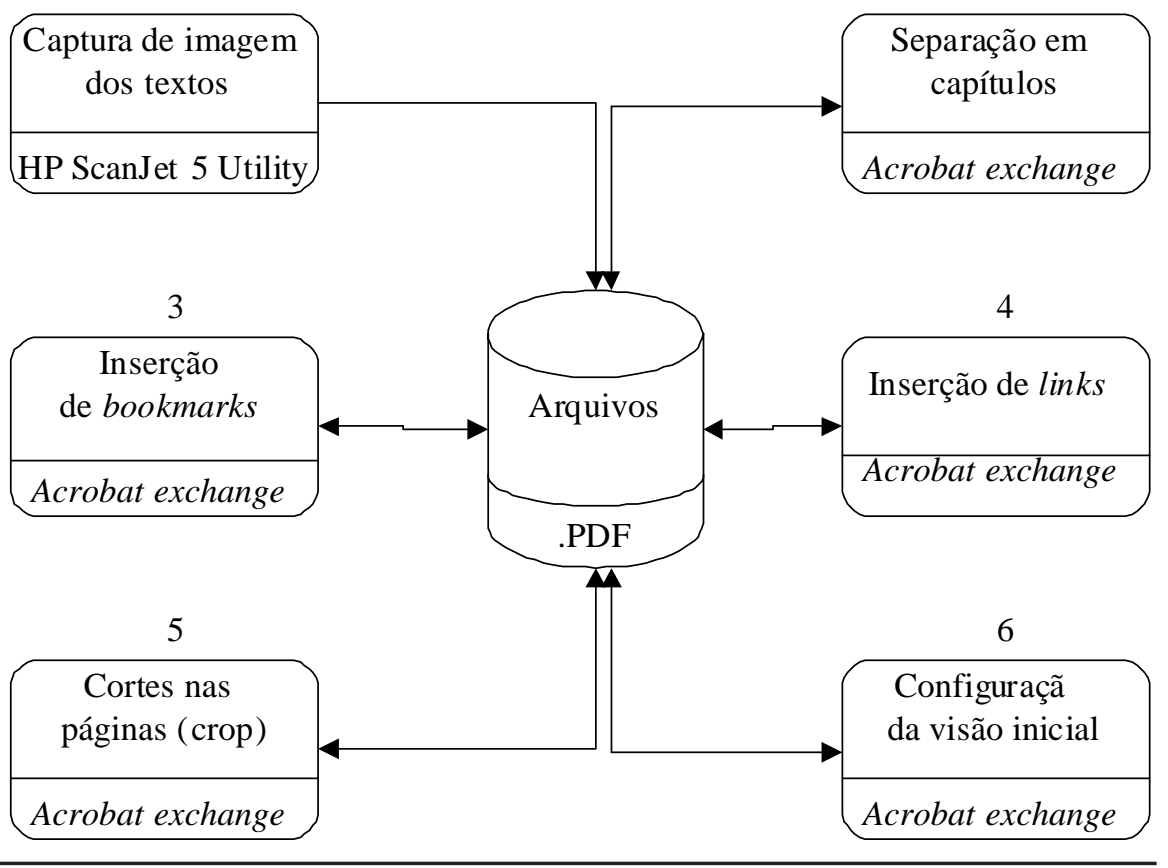

TABELA 5

Os tempos de realização das etapas do processo de digitalização

\section{ETAPAS}

TEMPO MÉDIO

Captura da imagens dos textos no scanner criação dos arquivos PDF (159 páginas)

Separação dos Arquivos em capítulos

16 minutos

Criação dos índices link no índice da obra

Criação das bookmarks

Cortes nas páginas (crop)

Configuração da visão inicial

Tempo médio para transformação de um texto do formato convencional (em papel) para o formato digital, segundo a

30 minutos

40 minutos*

30 minutos*

20 minutos*

1 minuto

137 minutos

(2 horas e $17 \mathrm{~min}$.) sistemática proposta (PDF) * Estas etapas são opcionais ao processo de digitalização e podem variar de acordo com as
características da obra. 
A conversão produziu um resultado considerado excelente. Sem maiores dificuldades, foi possível agrupar todas as partes que formavam a dissertação (arquivos de Word e Excel) em um mesmo arquivo no formato digital, sem preocupações maiores com as versões dos softwares utilizados.

A seguir, será apresentado o resumo esquemático do trabalho de conversão da dissertação de mestrado (figura 5).

São apresentados também os tempos médios verificados na execução de cada uma destas etapas (tabela 6).

\section{COMPARATIVO ENTRE AS SISTEMÁTICA APRESENTADAS}

Ao final deste trabalho, foram considerados positivos os resultados obtidos com a sistemática PDF, uma vez que, na comparação com a sistemática HTML pesquisada anteriormente, esta apresentou vantagens significativas conforme ilustra a tabela 7 . Os tempos apresentados são para um número padrão de 50 páginas e 12 figuras.

O principal ponto a favor da sistemática PDF é o tempo total demandado para transformação de maneira totalmente confiável, de uma obra em papel para o formato digital. Este ponto merece especial importância, pois torna viável a realização do processo em larga escala influenciando também na quantidade de recursos humanos necessários para estruturação de um núcleo para realização desta tarefa.

Segundo (Pohlmann ${ }^{5}$ ), no contexto do projeto de criação de uma biblioteca digital na PUCRS, está prevista a estruturação de um núcleo de digitalização de documentos. Este núcleo terá por objetivo a produção de acervo digital para a biblioteca digital da PUCRS, devendo contemplar os recursos necessários para a realização desta tarefa incluindo hardware, software, instalações e recursos humanos. Tais recursos humanos receberão treinamento e orientações a partir dos resultados apontados por esta pesquisa.
FIGURA 5

Esquema de conversão da dissertação de mestrado

\section{Conversão da dissertação de méstrado}

Documento digital na forma de texto para arquivo $P D F$

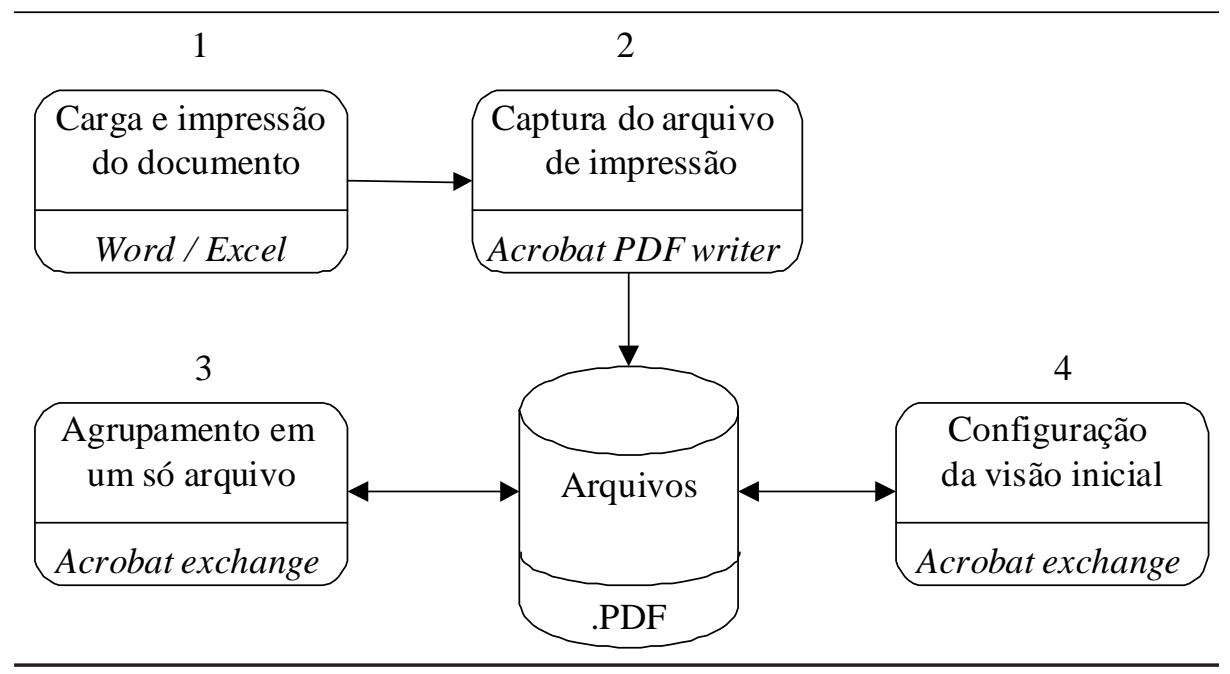

TABELA 6

Os tempos de realização das etapas do processo de conversão

\section{ETAPAS}

TEMPO MÉDIO

Carga e solicitação de impressão dos arquivos

10 minutos*

(1 Texto em MS-Word e 8 tabelas em MS-Excel)

Captura da Impressão e Conversão para PDF

10 minutos*

Agrupamento dos arquivos

15 minutos*

Configuração da visão inicial

1 minuto

Tempo total de conversão

36 minutos

TABELA 7

Comparativo entre a Sistemática HTML e as Sistemáticas PDF

\begin{tabular}{|c|c|c|c|}
\hline Característica & $\begin{array}{c}\text { Sistemática } \\
\text { HTML } \\
\text { Captura }\end{array}$ & $\begin{array}{l}\text { Sistemática } \\
\text { PDF - Captura } \\
\text { (imagem) }\end{array}$ & $\begin{array}{c}\text { Sistemática } \\
\text { PDF - Conversão } \\
\text { (texto) }\end{array}$ \\
\hline Mantém o leiaute original da obra & Não & Sim & Sim \\
\hline $\begin{array}{l}\text { Possibilidade de manipulação do } \\
\text { texto }\end{array}$ & Sim & Não & Sim \\
\hline $\begin{array}{l}\text { Possibilidade de realização de } \\
\text { pesquisas full-text }\end{array}$ & Sim & Não & Sim \\
\hline Espaço de armazenagem & Pequeno (texto) & $\begin{array}{c}\text { Aproxim. } \\
7 \text { vezes maior }\end{array}$ & $\begin{array}{c}\text { Aproxim. } \\
4 \text { vezes maior }\end{array}$ \\
\hline Revisão e correção do texto & *400 min & Não há & Não há \\
\hline Tempo de transmissão via rede & Baixo & 7 vezes maior & 4 vezes maior \\
\hline Tempo total aproximado de & $510 \mathrm{~min}$ & $46 \mathrm{~min}$ & $6 \mathrm{~min}$ \\
\hline
\end{tabular}

transformação de uma obra de

50 páginas e 12 figuras

\footnotetext{
* Observação: Cumpre salientar que o tempo relacionado a sistemática HTML foi determinado, com o trabalho sendo realizado por duas pessoas. Principalmente, a etapa de revisão e correção de texto pode ser agilizada agregando-se mais uma pessoa à equipe de trabalho.
} 
A seguir, apresenta-se uma sugestão de recursos mínimos necessários e os respectivos custos iniciais envolvidos para a formação de um núcleo de digitalização de documentos. Isto visa a permitir a comparação entre as sistemáticas apresentadas, levando em conta também a questão financeira. As sistemáticas de digitalização e conversão de documentos baseadas no formato PDF necessitam dos mesmos recursos e foram, por este motivo, agrupadas na mesma coluna. Os recursos indicados, bem como custos envolvidos, consideram a realidade da PUCRS.

Verifica-se que os valores diferem somente na aquisição das licenças dos software e ainda assim possuem valores aproximados. No entanto, deve-se realizar uma comparação relevando o custo relativo aos recursos humanos envolvidos na realização da tarefa nas diferentes sistemáticas, uma vez que a principal diferença identificada foi o tempo dispendido.

Utilizando o mesmo exemplo da tabela 7 , é possível verificar o custo dos recursos humanos envolvidos. Para tanto, foi calculado o valor da hora trabaIhada do profissional considerando 160 horas mensais $(\mathrm{R} \$ 2.255,90$ por profissional / 160 horas/mês $=R \$ 14,10 /$ hora por profissional)

Os pontos negativos da sistemática PDF são menos críticos para um projeto em larga escala, tais como o espaço de armazenagem, tempo de transmissão em rede, impossibilidade de realização de pesquisas full-text (somente para o caso da captura) e manipulação do texto.

A possibilidade de realização de conversões de documentos já existentes no formato digital para o formato PDF mostrou-se muito eficaz, em especial pelo fato de reproduzir o conteúdo dos documentos exatamente como estes seriam impressos e por facilitar a mesclagem de documentos, constituídos de diversos arquivos de software diferentes, gerando um só arquivo PDF.

\section{TABELA 8}

\section{Recursos para estruturação do núcleo}

Sistemáticas PDF

(Digitalização e Conversão)
Preço* Especificação Preço*
Sistemática HTML

\begin{tabular}{ll} 
Recurso & Especificação \\
\hline & 01 Computador: \\
& Pentium II $400 \mathrm{Mhz}$, \\
& 64 Mb RAM , HD 6,2 \\
Gb IDE, CD 24x, & Monitor 17", Placa de \\
Rede 10/100 Mbps, \\
Porta USB, Windows \\
NT 4.0 WS
\end{tabular}

Hardware

01 Scanner: HP ScanJet 6250: conexão USB, Bandeja ADF, Resolução 1200X 999.999 DPI

01 Licença Windows NT 4.0

Software

01 Licença Caere

Omni Page 8.0

01 Aparelho de $\mathrm{Ar}$ Condicionado 18.000 btus

02 mesas para microcomputadores

Instalações

02 luminárias de 03

lâmpadas com

refletores

02 pontos de rede

02 cadeiras com rodízios

Recursos 02 Remuneração com

Humanos Encargos Sociais (Bibliotecário Júnior)

Total

\footnotetext{
Os valores de
}

$14.604,31$
01 Computador:

Pentium II $400 \mathrm{Mhz}$,

64 Mb RAM , HD 6,2

6.158,00 Gb IDE, CD 24x, Monitor 17", Placa de

Rede 10/100 Mbps,

Porta USB, Windows

NT 4.0 WS

01 Scanner: HP

ScanJet 6250: conexão

1.598,00 USB, Bandeja ADF,

Resolução 1200X

999.999 DPI Incluída no 01 Licença Windows
computador NT 4.0

$6.158,00$

(158,00

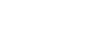

$1.598,00$

(.5.00

Incluída no computador

500,00

549,00 01 Licença Adobe Acrobat 3.01

1.177,51 01 Aparelho de $\mathrm{Ar}$ Condicionado 18.000 btus

188,00 02 mesas para microcomputadores

$1.177,51$

100,00 02 luminárias de 03 lâmpadas com refletores

166,0002 pontos de rede

166,00

156,00 02 cadeiras com rodízios

156,00

02 Remuneração com

4.511,80 Encargos Sociais

(Bibliotecário Júnior)

$4.511,80$

* Os valores de referência estão em Reais, cotados na época a 1,71 em relação ao dólar americano

TABELA 9

Comparativo entre despesas com recursos humanos

\begin{tabular}{lccc}
\hline & $\begin{array}{c}\text { Sistemática } \\
\text { HTML Captura }\end{array}$ & $\begin{array}{c}\text { Sistemática } \\
\text { PDF - Captura } \\
\text { (imagem) }\end{array}$ & $\begin{array}{c}\text { Sistemática } \\
\text { PDF - Conversão } \\
\text { (texto) }\end{array}$ \\
\hline Tempo dispendido & $510 \mathrm{~min}$ & $46 \mathrm{~min}$ & $6 \mathrm{~min}$ \\
Cálculo & $\begin{array}{c}\mathrm{R} \$ 14.10 \times 8.5 \\
\text { horas } \times 2 \text { pessoas }\end{array}$ & $\begin{array}{c}\mathrm{R} \$ 14.10 \times 0.76 \\
\text { horas } X 2 \text { pessoas }\end{array}$ & $\begin{array}{c}\mathrm{R} \$ 14.10 \times 0.1 \text { horas } \\
\times 2 \text { pessoas }\end{array}$ \\
$\begin{array}{l}\text { Despesa com } \\
\text { recursos humanos }\end{array}$ & $\mathrm{R} \$ 239,70$ & $\mathrm{R} \$ 21,43$ & $\mathrm{R} \$ 2,82$ \\
\hline
\end{tabular}


Tais características indicam maior facilidade de formação de um acervo contendo documentos recentes (que teoricamente já existem em meio digital), possibilitando inclusive a realização de pesquisas full-text nos arquivos que foram convertidos a partir do formato texto, além de favorecer a padronização das publicações digitais e, por conseqüência, o posterior armazenamento, recuperação e manipulação.

Deve-se registrar que os problemas que impõem dificuldades à realização do OCR, sejam eles causados pelo estado de conservação do acervo, tais como manchas, amassados, riscos e anotações, ou ligados a ineficiência dos softwares de OCR para tratamento de características, como fórmulas matemáticas, figuras, trechos manuscritos, letras muito pequenas ou borradas, podem ser contornados pela digitalização utilizando a sistemática PDF. Para tanto, basta que as obras sejam digitalizadas como imagens, e será possível realizar a leitura das mesmas, por meio de um arquivo PDF, conforme a aparência original no momento da digitalização.

Neste sentido, é importante que se desenvolvam ferramentas para realização de tratamento óptico nos arquivos de imagens PDF, que filtrem automaticamente as características indesejáveis - adulterações, manchas, amassados entre outros -, melhorando a aparência das obras.

\section{CONSIDERAÇÕES FINAIS}

Inicialmente, os trabalhos de construção do acervo da Biblioteca Digital da PUCRS serão realizados com a utilização do software Adobe Acrobat para digitalização das obras existentes no formato tradicional (papel) e também para conversão dos documentos já existentes em um formato digital diferente do HTML. Os documentos que já estiverem no formato HTML serão mantidos, pois este formato permite a realização de pesquisas full-text, possui tamanho inferior ao PDF e atende às diretrizes definidas para este trabalho apontadas no item Diretrizes de Trabalho.
A disponibilização das obras na Internet será feita, em primeiro momento, pelo software ALEPH, que gerencia a catalogação e consulta do acervo da Biblioteca Central.

O ALEPH possui uma interface que permite a realização de consultas pela Internet, possibilitando aos usuários verificar a existência das obras no acervo, bem como a sua disponibilidade para empréstimo. Os recursos de pesquisa do ALEPH baseiam primariamente em autores, títulos e assuntos, mas é possível realizar consultas avançadas acessando qualquer informação constante no registro de cadastramento da obra. Pode-se ainda combinar diversos argumentos de pesquisa mediante a utilização de lógica booleana.

No caso de as obras já existirem no formato digital, o ALEPH fornece um link para acesso ao documento na íntegra, permitindo assim que o usuário possa ler a obra digital pela Internet, sem precisar se deslocar até a biblioteca.

As pesquisas full-text serão realizadas com recursos de pesquisa do Adobe Acrobat Reader, uma vez que o ALEPH não consegue indexar os textos existentes no formato PDF. No entanto, para acessar os recursos de pesquisa full-text, o usuário deve realizar o download do documento (copiá-lo para sua máquina) e abri-lo por meio do Adobe Acrobat Reader, pois a consulta ao arquivo PDF realizada com auxílio do browsernão oferece o recurso de pesquisa full-text .

Com o crescimento do acervo digital, torna-se necessário a utilização de outros softwares que possuam recursos mais adequados à recuperação e acesso a este acervo, permitindo a utilização de técnicas avançadas de pesquisa com a utilização de linguagem natural, utilização de parâmetros fonéticos e técnicas de inteligência artificial. Para tanto, está prevista a utilização do software IBM Digital Library, que recentemente foi disponibilizado e encontrase em fase de instalação em nosso laboratório.
Como prosseguimento deste trabalho, será realizado, em conjunto com a Faculdade de Biologia e com o suporte da Biblioteca Central, a digitalização de obras de formatos diversos tais como fotografias, sons, textos e imagens. Esses recursos serão utilizados para a elaboração de materiais didáticos, servindo como fonte de pesquisa para a avaliação das características do Adobe Acrobat Reader para suporte a estas mídias, comparando-as com a utilização da linguagem HTML para o mesmo propósito.

A continuidade deste trabalho objetiva definir novas sistemáticas que sejam adequadas à digitalização de obras e materiais existentes nos mais diversos formatos, servindo assim para orientar a estruturação do núcleo de digitalização de documentos e produção de materiais digitais de cunho didático. 


\section{REFERÊNCIAS BIBLIOGRÁFICAS}

1. POHLMANN, Omer F. Campos, Márcia B. Raabe, André L. John, Fabiana. Viera, Sônia. Em Direção a Criação de uma Biblioteca Digital na Pontifícia Universidade Católica do Rio Grande do Sul: - Uma experiência Prática. II Seminário Internacional de Bibliotecas associadas a UNESCO, Cienfuegos Cuba. 23 a 27 de maio de 1998.

2. HAIGH, Susan. Optical Character Recognition (OCR) as a Digitization Technology. [Citado em 10 jan. 1998]. Disponível em WWW: [http://collection.nlc-bnc.ca/100/ $201 / 301 /$ netnotes/netnotes-h/ notes37.htm]

3. CAERE Corporation. A Quantum Leap in Accuracy. [Citado em 11 jan. 1998]. Disponível em WWW: [http:// www.caere.com/live/content/products/ amaretto/amaretto.htm]

4. ADOBE Acrobat 3.0 Product Information. [Citado em 14 jul. 1998]. Disponível em WWW: [http://www.adobe.com/prodindex/ Acrobat/prodinfo.html]

5. POHLMANN, Omer F. Raabe, André L. Direito Autoral no Contexto de Bibliotecas Digitais. III Congresso Internacional de (Tele) Informática Educativa, Santa Fe Argentina. 14 a 17 de abril de 1999.

\section{Comparative study between systematics of digitisation of documents: Formats HTML and PDF}

\section{Abstract}

This article presents the resulting experience of Digital Library Group of PUCRS University, for the process of capture and conversion of existing documents from traditional format (paper) to a digital format. The major steps of the process are presented and evaluated using two different systematics: one based on HTML conversion; and other based on the creation of PDF files for Adobe Acrobat Reader software.

Critical issues such as Optical Character Recognition (OCR) and characteristics evaluation of the collection to be converted are approached also.

At the end, is presented a comparative study between the two systematics, identifying positive and negative characteristics to be considered for choosing a work direction.

\section{Keywords}

Conversion of documents from the traditional to the digital format; Systematics of conversion to HTML; Creation of PDF files; OCR technologies.

\section{André Luís Alice Raabe}

Bacharel em Informática, PUCRS, 1997. Mestrando em Informática, PUCRS 1998.

\section{Omer Pohlmann Filho}

Bacharel em Administração de Empresas, PUCRS, 1979. Especialista em Análise de Sistemas, PUCRS, 1981. Mestre em Informática, PUCRS, 1996.

\{araabe,omer\}@cglobal.pucrs.br 